\title{
Formation of Nanopatterned Polymer Blends in Photovoltaic Devices
}

\author{
Ximin He, ${ }^{\dagger,}$ ₹ Feng Gao, ${ }^{\S}$ Guoli Tu, ${ }^{\ddagger}$ David Hasko, ${ }^{\S}$ Sven Hüttner, ${ }^{\S}$ Ullrich Steiner,, ,II \\ Neil C. Greenham, ${ }^{*}$, Richard H. Friend, ${ }^{\S *}$ and Wilhelm T. S. Huck* ${ }^{*}, \neq, \perp$
}

\begin{abstract}
${ }^{\dagger}$ The Nanoscience Centre, University of Cambridge, $11 \mathrm{~J}$. J. Thomson Avenue, Cambridge CB3 OFF, United Kingdom, ${ }^{*}$ Melville Laboratory, Department of Chemistry, University of Cambridge, Lensfield Road, Cambridge CB2 1EW, United Kingdom, ${ }^{\S}$ Cavendish Laboratory, Department of Physics, University of Cambridge, J. J. Thomson Avenue, Cambridge CB3 OFF, United Kingdom, "Freiburg Institute for Advanced Studies (FRIAS) University of Freiburg, D-79104 Freiburg, Germany, and ${ }^{\perp}$ Radboud University Nijmegen, Institute for Molecules and Materials, Heyendaalseweg 135, 6525 AJ Nijmegen, The Netherlands
\end{abstract}

\begin{abstract}
In this paper, we demonstrate a double nanoimprinting process that allows the formation of nanostructured polymer heterojunctions of composition and morphology that can be selected independently. We fabricated photovoltaic (PV) devices with extremely high densities $\left(10^{14} / \mathrm{mm}^{2}\right)$ of interpenetrating nanoscale columnar features in the active polymer blend layer. The smallest feature sizes are as small as $25 \mathrm{~nm}$ on a $50 \mathrm{~nm}$ pitch, which results in a spacing of heterojunctions at or below the exciton diffusion length. Photovoltaic devices based on double-imprinted poly((9,9-dioctylfluorene)-2,7-diyl-alt-[4,7-bis(3-hexylthien-5-yl)-2,1,3-benzothiadiazole]-2',2"'-diyl) (F8TBT)/ poly(3-hexylthiophene) (P3HT) films are among the best polymer-polymer blend devices reported to date with a power conversion efficiency (PCE, $\eta_{\mathrm{e}}$ ) of $1.9 \%$
\end{abstract}

KEYWORDS Polymer photovoltaics, nanoscale morphology, nanoimprint lithography, polymer blends

$\bigcirc^{\text {in }}$ ince the first reports on polymer bulk heterojunction (BHJ) devices, the importance of an interpenetrating, nanoscale network morphology of phase-separated donor and acceptor materials has been recognized. ${ }^{1,2}$ Such structures provide the spatially distributed interfaces necessary for efficient charge separation of the photogenerated excitons and direct pathways for collecting electron and holes. Current device fabrication is based on spincoating and annealing procedures, which result in highly irregular, irreproducible morphologies that strongly depend on processing conditions and materials used. ${ }^{3-7}$ The efficiency of the phase-separated interpenetrating donor-acceptor results from the ability to efficiently dissociate photogenerated excitons and transport the dissociated charges to the respective electrodes. Since the first reports on polymer blend devices, device efficiencies have increased considerably through improvements in materials, processing, and control over the morphology. Sustained efforts have improved polymer PV devices based on fullerene derivatives in combination with electron-donating polymers such thiophene polymers to efficiencies $\eta_{\mathrm{e}}$ in the range of $4-7 \% .^{8-11}$ Allpolymeric devices would overcome the low extinction coefficient and reduced open-circuit voltage associated with fullerenes and allow the use of a much wider range of materials. Although much less research has been devoted

* To whom correspondence should be addressed. E-mail: (N.C.G.) ncg11@ cam.ac.uk; (R.H.F.)rhf10@cam.ac.uk; (W.T.S.H.)wtsh2@cam.ac.uk.

Received for review: 12/11/2009

Published on Web: 03/03/2010 to all-polymer PV devices, promising improvements in efficiency to around $1.8 \%$ have been reported ${ }^{12-14}$ with a very recent report on blends of poly(phenylthiophene) and poly[(cyanovinylene)phenylene] derivatives even reaching $2.0 \% \cdot{ }^{15}$ To further improve the efficiency of all-polymeric $\mathrm{BHJ}$ devices, the internal interfacial area must be as large as possible, while continuous pathways for the dissociated charges to reach the electrodes must be maintained. For spin-coated polymer blends, this means that phase separation needs to be arrested at the nanometer scale as the exciton diffusion length is in the order of $10 \mathrm{~nm}$ and micrometer phase separation significantly diminishes device performance. ${ }^{3,7}$ Even when this is achieved, the randomly interspersed electron donor and acceptor phases can lead to charge trapping, ${ }^{16}$ or incomplete photoluminescence (PL) quenching in the regions of conjugated polymer that are more than an exciton diffusion length away from an acceptor. ${ }^{17}$ Spin-coating procedures involving highly volatile solvents have been developed to optimize the phase separation process. ${ }^{18-20}$ However, the morphologies formed in spincoated films are very strongly dependent on processing conditions (spin speed, solvent, temperature, humidity, concentration) as well as the materials used. The lack of control in device fabrication greatly hampers the systematic study of the properties of demixed blend PV devices and restricts their exploitation in large-area solar cells.

Here, we present a completely new approach to nanopatterned polymer blends through nanoimprint lithography (NIL), ${ }^{21}$ as shown schematically in Figure 1. A detailed description of the imprinting method, as well as details on 


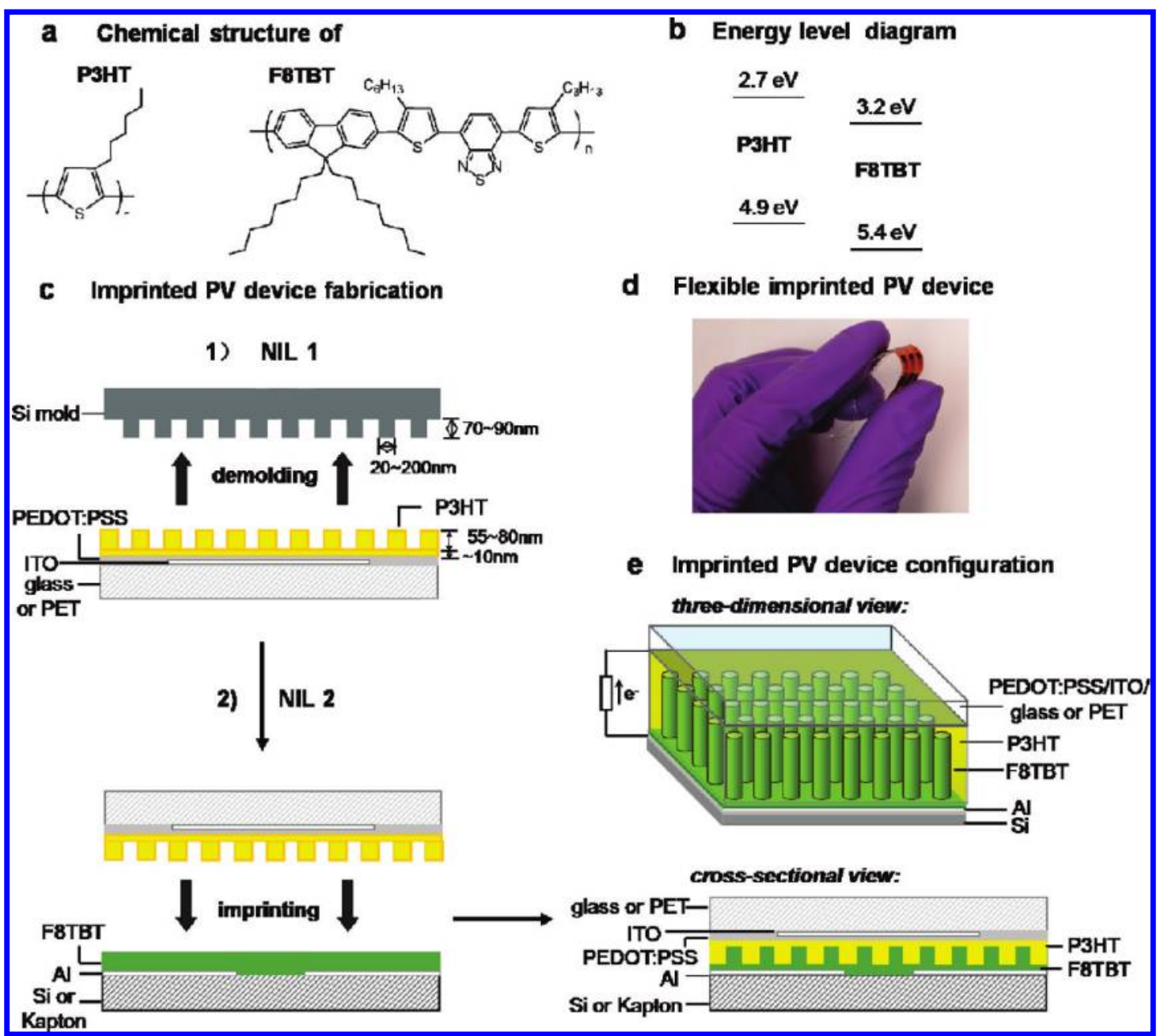

FIGURE 1. (a) Chemical structure and (b) energy level of P3HT and F8TBT. (c) Schematic procedure of imprinted PV device fabrication. (1) Patterning of P3HT film spin-cast on a ITO/glass substrate coated with PEDOT/PSS as anode by SANIL using a Si mold. (2) Using the patterned P3HT as a mold to imprint a F8TBT film spin-cast on a Al cathode on a Si wafer or Kapton substrate, resulting in a double-imprinted PV device. (d) A photograph of flexible double-imprinted P3HT/F8TBT PV devices with six $2 \times 4 \mathrm{~mm}$ pixels based on PET and Kapton polyimide films. (e) Three-dimensional and cross-sectional view of the imprinted PV device configuration.

the materials used, is given in the Supporting Information. First, a spin-coated film of the electron donor polymer P3HT on PEDOT/PSS-coated ITO/glass or poly(ethylene terephthalate) (PET) substrate was patterned in a first nanoimprinting step. As P3HT is easily oxidized and degrades at elevated temperatures (above $60^{\circ} \mathrm{C}$ ), we employed solvent-vaporassisted nanoimprint lithography (SANIL) ${ }^{22}$ under $\mathrm{N}_{2}$ flow and imprinted at room temperature and low pressure (for full experimental details see Supporting Information). Prior to demolding and the subsequent imprinting of F8TBT films, the patterned P3HT films were thermally annealed to improve the crystallinity and mechanical strength. The crucial part of our procedure is to use the first patterned film as a stamp in the second imprinting step of the acceptor polymer F8TBT, which was spin-coated on a thermally evaporated Al cathode on a Si wafer or Kapton polyimide film. Hence, upon double imprinting a functional photovoltaic device based on a bilayer polymer blend film with precisely defined interface geometry was formed. This approach leads to precisely defined, interdigitated polymer bilayers with structures at the $25 \mathrm{~nm}$ level. Furthermore, the anode is completely covered with the electron donor material and likewise the cathode is covered with the electron acceptor polymer. There is therefore a direct pathway for all charges generated. It should be noted that our method is rather different from initial reports in the literature on PV devices containing imprinted P3HT. ${ }^{23-25}$ These studies used relatively large imprinted features and electron accepting small molecules (PCBM, perylene) that were deposited either on the initial film after cross-linking, or via vapor deposition. Our method, which is based on a manufacturable and scalable technology, ${ }^{26}$ is suitable for any polymer combination with a difference in $T_{\mathrm{g}}$, regardless of interaction parameter $\chi$, solubility properties, and molecular weights.

To perform a systematic study of the influence of feature size and interface area on PV device performance, a series of masters were either obtained commercially from NIL Technology ApS or fabricated in-house using e-beam lithog- 


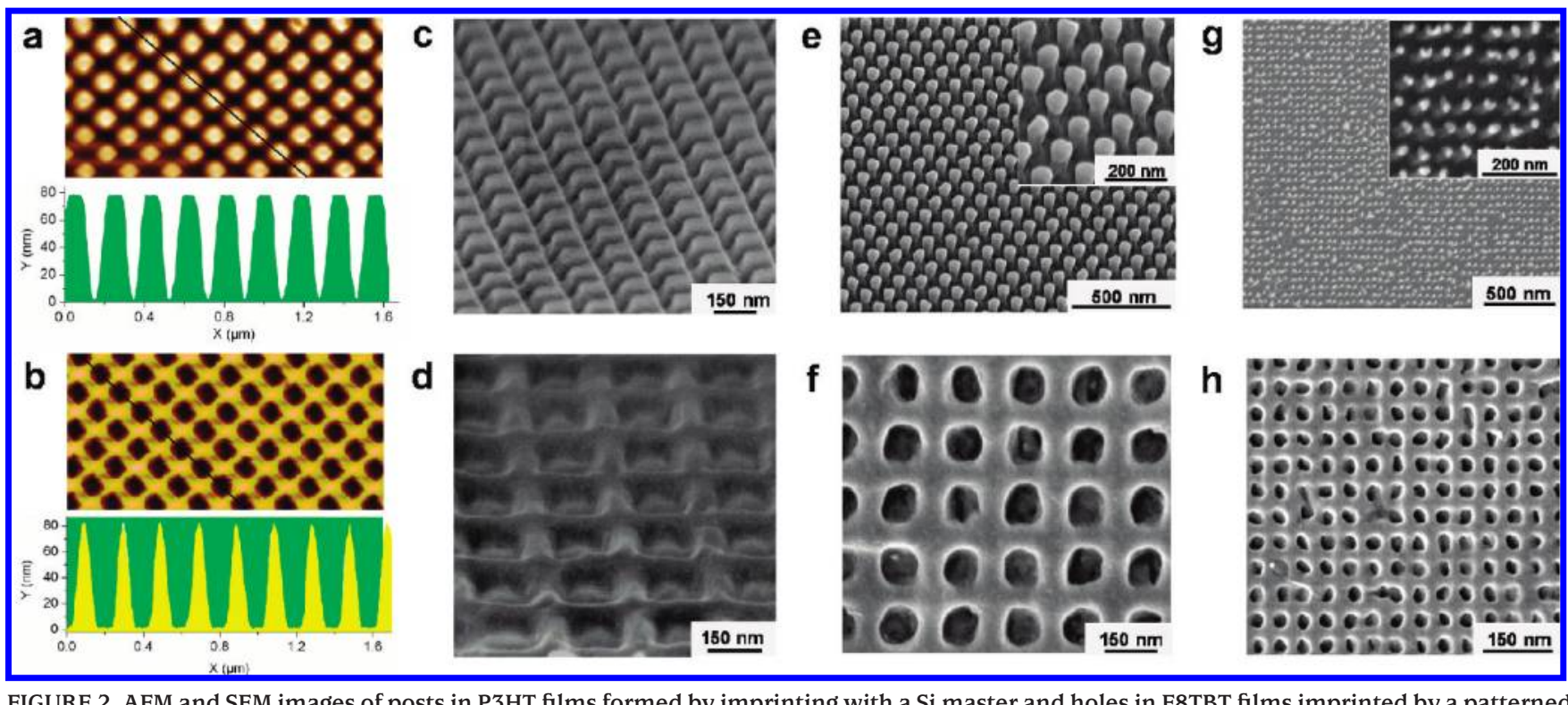

FIGURE 2. AFM and SEM images of posts in P3HT films formed by imprinting with a Si master and holes in F8TBT films imprinted by a patterned P3HT film. (a) AFM images and lines traces of $100 \mathrm{~nm}$ wide posts in P3HT film and (b) $100 \mathrm{~nm}$ wide holes in F8TBT. The line trace in (d) shows the exact replication of the P3HT features into the F8TBT film. (c,d) SEM image of samples shown in (a) and (b), respectively. (e-h) SEM images of $80 \mathrm{~nm}(\mathrm{e}, \mathrm{f})$ and $25 \mathrm{~nm}(\mathrm{f}, \mathrm{h})$ posts in P3HT and holes in F8TBT.

raphy. The molds contain 75-80 $\mathrm{nm}$ deep 2D dot patterns with feature sizes of 200,150,100, 80, 40, and $25 \mathrm{~nm}$ and equivalent spacing (i.e., pitch is double the feature size). The total imprinted P3HT/F8TBT area was $4 \times 2 \mathrm{~mm}^{2}$ for all patterns, forming 6 pixels of PV devices in each double imprinting experiment.

To investigate the internal structure of the P3HT/F8TBT interface, the two layers were either physically prised apart or the F8TBT layer was dissolved in a selective solvent $\left(\mathrm{CH}_{2} \mathrm{Cl}_{2}\right)$ to reveal the imprinted P3HT surfaces for SEM and AFM measurements (Figure 2). In general, all features over
$80 \mathrm{~nm}$ are nearly perfectly replicated over the whole area of the stamp. The high (>3:1) aspect ratio of the $25 \mathrm{~nm}$ dots leads to some distortion in the P3HT pillars and the F8TBT holes are not uniform. Furthermore, Figure 2c, d shows that the features in P3HT and F8TBT are exact replicas of each other, confirming that the imprinted polymer film acts as a robust stamp in the second imprinting step.

Devices. Previous studies have shown that only annealed blends of P3HT and F8TBT produced efficient photovoltaic devices. ${ }^{11}$ A detailed study using optical absorption and photoluminescence spectroscopy revealed that the device
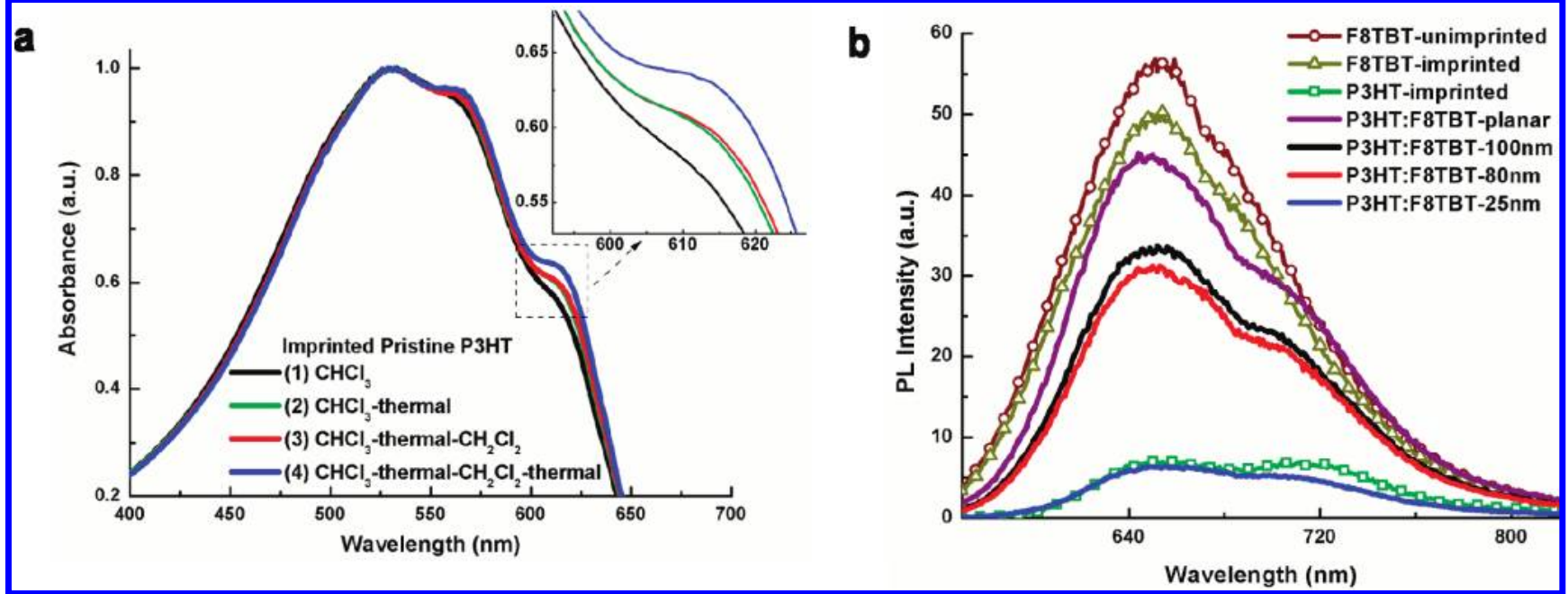

FIGURE 3. (a) Absorption spectra for a P3HT film treated by solvent-vapor and thermal annealing. Black curve, as-cast plain film; red curve, $\mathrm{CHCl}_{3}$ vapor annealed nanopatterned film formed by SANIL; green curve, $\mathrm{CHCl}_{3}$ and subsequently $\mathrm{CH}_{2} \mathrm{Cl}_{2}$ vapor annealed nanopatterned film; blue curve, as green, followed by thermal annealing. (b) Photoluminescence spectra of films of pure P3HT (an imprinted film by Si mold) and pure F8TBT (a nonimprinted pristine film and an imprinted film by patterned P3HT followed by removal of P3HT) as well as imprinted P3HT/F8TBT with planar and 100, 80, and $25 \mathrm{~nm}$ patterned interface. The PL intensities were measured under identical excitation and collection conditions and corrected for the absorbance of the different films. 
TABLE 1. Summary of Device Performance of P3HT/F8TBT Photovoltaic Devices

\begin{tabular}{|c|c|c|c|c|c|c|c|}
\hline & $\begin{array}{c}\text { feature } \\
\text { size-width } \\
\quad(n m)\end{array}$ & $A / A_{0}{ }^{a}$ & $\begin{array}{c}\max \\
\text { EQE }(\%)\end{array}$ & $\begin{array}{l}V_{\mathrm{oc}} \\
(\mathrm{V})\end{array}$ & $\begin{array}{c}J_{\mathrm{sc}} \\
\left(\mathrm{mA} / \mathrm{cm}^{2}\right)\end{array}$ & FF & $\begin{array}{l}\text { PCE } \\
(\%)^{b}\end{array}$ \\
\hline planar & & 1 & 10.2 & 1.06 & 0.94 & 0.39 & 0.36 \\
\hline patterned & 200 & 1.45 & 16.8 & 1.08 & 1.81 & 0.39 & 0.77 \\
\hline 2D dot arras & 150 & 1.60 & 17.8 & 1.12 & 1.99 & 0.39 & 0.87 \\
\hline (width $=\mathrm{sp}$ & 100 & 1.90 & 19.0 & 1.14 & 1.96 & 0.43 & 0.97 \\
\hline & 80 & 2.00 & 20.6 & 1.14 & 2.35 & 0.43 & 1.14 \\
\hline & 40 & 3.67 & 23.8 & 1.13 & 2.79 & 0.47 & 1.48 \\
\hline & 25 & 4.20 & 26.1 & 1.14 & 3.30 & 0.49 & 1.85 \\
\hline blend & & & 19.7 & 1.12 & 2.32 & 0.42 & 1.09 \\
\hline
\end{tabular}

${ }^{a} A / A_{0}$ is donor/acceptor interface area ratio of patterned to planar-interface PV devices. ${ }^{b}$ Illumination intensity equivalent to $100 \mathrm{~mW} \mathrm{~cm}^{-2}$ after spectral mismatch correction using AM $1.5 \mathrm{G}$ solar simulator.

efficiency could be attributed to an increase in the dissociation efficiency of bound electron-hole pairs resulting from improved carrier mobilities in ordered materials. ${ }^{27}$ During the fabrication of the double-imprinted PV cells, the P3HT film was exposed to chloroform $\left(\mathrm{CHCl}_{3}\right)$ and dichoromethane $\left(\mathrm{CH}_{2} \mathrm{Cl}_{2}\right)$ while both the P3HT and F8TBT films underwent thermal annealing during the second imprinting step, fol- lowed by a post imprinting annealing step $\left(120^{\circ} \mathrm{C}\right.$ for 5 min). As seen in Figure 3a, a characteristic shoulder centered at $\sim 610 \mathrm{~nm}$ was observed in the UV-vis absorption spectra of P3HT, the intensity of which is correlated with the degree of crystallinity of P3HT. The shoulder increases significantly upon thermal annealing, but is already present directly after SANIL and this might be attributed to the effect of nanoconfinement in the imprinting mold, which can enforce order in rigid, (liquid) crystalline polymers. ${ }^{28,29}$

PL measurements (Figure 3b) showed strong quenching in imprinted P3HT/F8TBT films and a decrease of PL intensity as pattern feature size decreased. The imprinting process itself led to only a small (10\%) reduction in the PL intensity of the F8TBT film after double-imprinting and subsequent removal of the P3HT layer. Very strong PL quenching (by $87 \%$ in comparison to imprinted F8TBT at $651 \mathrm{~nm}$ ) was observed in the double imprinted films with $25 \mathrm{~nm}$ features, strongly indicating that these dimensions are similar to or smaller than the exciton diffusion length in both materials. Indeed, the residual emission appears to be coming from P3HT, suggesting that the F8TBT emission is almost completely quenched. Interestingly, the PL intensities

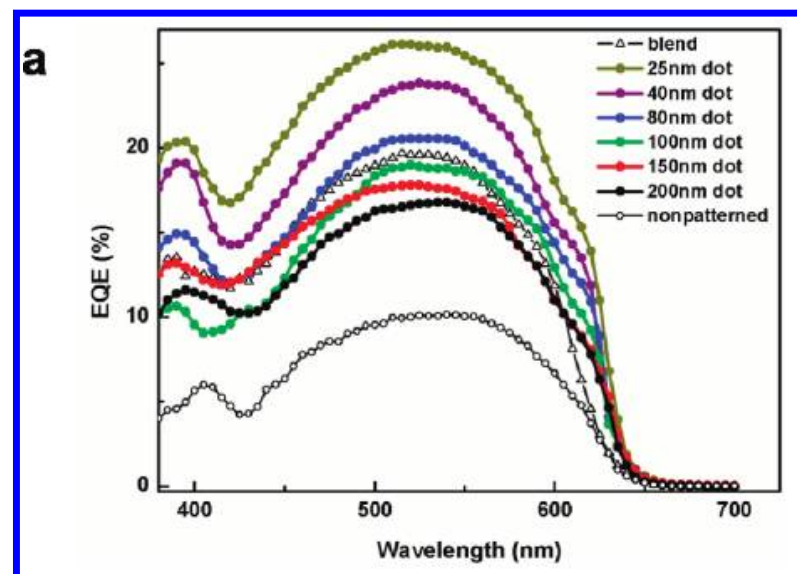

b
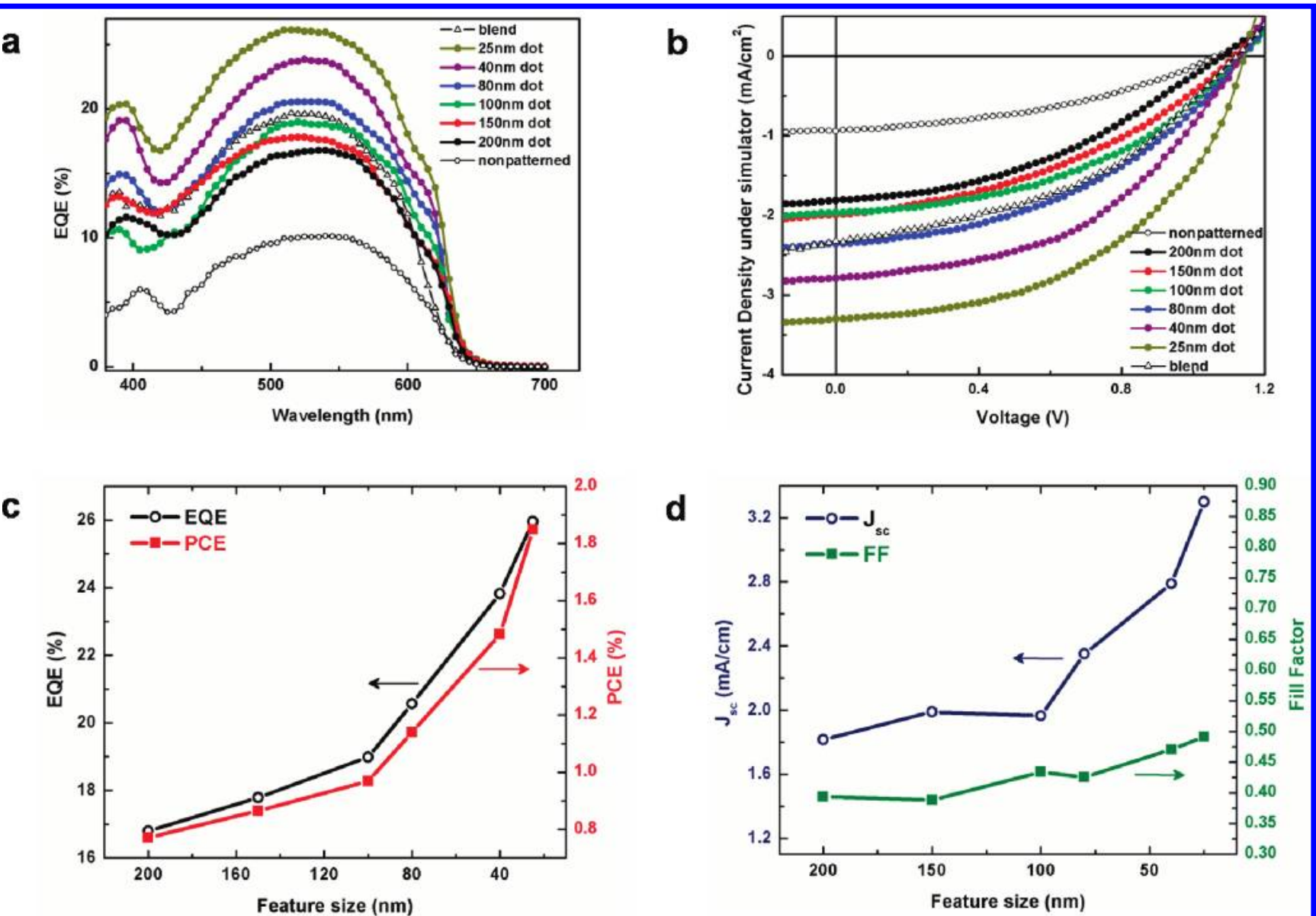

FIGURE 4. (a) EQE and (b) $J-V$ characteristics of the double-imprinted PV cells for a series of feature sizes in comparison to planar and blend controls under solar illumination conditions. The correlation of pattern feature sizes with (c) EQE (low intensity) $(-\mathrm{O}-)$ and PCE $(-\square-$, red), and (d) $J_{\mathrm{sc}}(-\mathrm{O}-)$ and $\mathrm{FF}(-\mathbf{-}-$, green). 
of the double imprinted films containing 100 and $80 \mathrm{~nm}$ features were also strongly affected (only 67 and $62 \% \mathrm{PL}$ remaining at $651 \mathrm{~nm}$, respectively). These results are consistent with an F8TBT exciton diffusion range of $10 \mathrm{~nm}$. By changing the imprint pattern, double imprinting has the potential to allow the dimensions of both phases to be independently tailored to match the respective exciton diffusion length in either phase. This is a key advantage of the technique and it might help in identifying new acceptor materials that are not based on fullerenes.

Table 1 presents a concise summary of the main performance parameters of nanostructured solar cells. All imprinted cells exhibited a high open circuit voltage $\left(V_{\mathrm{OC}}\right)>1.1$ $\mathrm{V}$, comparable to the blend control device fabricated under the same conditions as previously reported. ${ }^{24}$

Figure 4 shows the significant increase of the external quantum efficiency (EQE) with decrease in feature sizes with the spincoated polymer blend performing roughly equal to the 80 and $100 \mathrm{~nm}$ imprinted films. The current densityvoltage $(J-V)$ characteristics show significant increases in both short circuit current density ( $\mathrm{J}_{\mathrm{s}}$ ) with decreasing feature sizes. The $J-V$ curve of the blend control device overlaps with that of the $40 \mathrm{~nm}$ featured imprinted one, although the latter shows an improved fill factor (FF).

A clear improvement in the PCE of the nanostructured devices is observed with the $25 \mathrm{~nm}$ features leading to a PCE of $1.85 \%$, which is $50 \%$ higher than the blend control device. It should be noted that the first reported P3HT/F8TBT blends quoted efficiencies of $1.8 \%$ that might be attributed to a higher grade F8TBT; a more reproducible value for the efficiency of blend devices is around $1 \% .{ }^{11,25}$ The most notable influence of nanopatterning on device performance can be seen in the fill-factor, which increases to the highest reported value for all-polymer devices (0.49) for the $25 \mathrm{~nm}$ features. Previous work on modeling of the behavior of nanostructured organic photovoltaic devices, ${ }^{30}$ identified a number of possible reasons for an improved FF. We suggest that improved hole charge carrier mobility and better geminate pair dissociation in patterned devices contribute to the improved FF. In a blend device, geminate pair separation might be impeded by the intermixed morphology, whereas in the imprinted structures (as in bilayer devices) geminate pairs have more direct pathways to charge separation. Further experiments will be required to verify the origin of the high fill factors observed in the imprinted devices. Furthermore, the double imprinting process ensures that both the donor and acceptor polymer are completely pure, whereas spincoated blends not always fully phase-separate, leading to suboptimal charge mobilities. Finally, we note that in spincoated devices, it is usually impossible to avoid wetting of the "wrong" polymer at either electrode, ${ }^{5}$ whereas our devices have pure polymer layers adjacent to the appropriate electrodes, preventing diffusion of carriers to the wrong electrode.
Conclusion. In summary, we have shown that this novel imprinting method produces the best performance yet reported for a polymer-polymer system and this improvement results primarily through the improved fill factor over that obtained for solution demixed devices. We consider that this is due to the avoidance of locally trapped photogenerated charges due to the poorly controlled morphology formed from the demixed polymer blend. The absolute efficiency of our devices is limited by the $80 \mathrm{~nm}$ height of the imprinted structure as shown in Figure 2a. Current devices only absorb $58 \%$ of the available solar intensities between 375 and $640 \mathrm{~nm}$ (based on optical modeling of the device structure). Hence there is considerable room for improvement in power conversion efficiency if the device thickness can be increased while retaining efficient charge separation and collection. Our work indicates that charge separation at polymer-polymer heterojunctions can be similarly effective to polymerfullerene systems, and demonstrates that there is scope to find a broader range of electron acceptors beyond the widely used fullerenes that can provide high PV efficiency when the heterojunction morphology is controlled appropriately.

Acknowledgment. This work was supported by the Engineering and Physical Science Research Council. X.H. was supported by the Gates Cambridge Trust.

Supporting Information Available. Materials, device fabrication, and device testing. This material is available free of charge via the Internet at http://pubs.acs.org.

\section{REFERENCES AND NOTES}

(1) Halls, J. J. M.; Walsh, C. A.; Greenham, N. C.; Marseglia, E. A.; Friend, R. H.; Moratti, S. C.; Holmes, A. B. Nature 1995, 376, 498.

(2) Yu, G.; Gao, J.; Hummelen, J. C.; Wudl, F.; Heeger, A. I. Science 1995, 270, 1789

(3) Coakley, K. M.; McGehee, M. D. Chem. Mater. 2004, 16, 4533.

(4) Hoppe, H.; Sariciftci, N. S. L. Mater. Chem. 2006, 16, 45.

(5) Yang, X.; Loos, J. Macromolecules 2007, 40, 1353.

(6) Coakley, K. M.; Liu, Y.; Goh, C.; McGehee, M. D. MRS Bull. 2005, $30,37$.

(7) Günes, S.; Neugebauer, H.; Sariciftci, N. S. Chem. Rev. 2007, 107, 1324.

(8) Reyes, R. R.; Kim, K.; Carroll, D. L. Appl. Phys. Lett. 2005, 87, No. 083506.

(9) Kim, J.; Kim, S.; Lee, H.; Lee, K.; Ma, W.; Huong, X.; Heeger, A. L. Adv. Mater. 2006, 18, 572.

(10) Park, S. H.; Roy, A.; Beaupre, S.; Cho, S.; Coates, N.; Moon, J. S.; Moses, D.; Leclerc, M.; Lee, K.; Heeger, A. I. Nat. Photon. 2009, 3, 297.

(11) Chen, H. Y.; Hou, J.; Zhang, S.; Liang, Y.; Yang, G.; Yang, Y.; Yu, L.; Wu, Y.; Li, G. Nat. Photon. 2009, 3, 649.

(12) McNeill, C. R.; Abrusci, A.; Zaumseil, J.; Wilson, R.; McKiernan, M. J.; Burroughes, J. H.; Halls, J. J. M.; Greenham, N. C.; Friend, R. H. App. Phys. Lett. 2007, 90, 193506.

(13) Kietzke, T.; Horhold, H. H.; Neher, D. Chem. Mater. 2005, 17, 6532.

(14) Veenstra, S. C.; Loos, J.; Kroon, J. M. Proq. Photovolt 2007, 15, 727 .

(15) Holcombe, T. W.; Woo, C. H.; Kavulak, D. F. J.; Thompson, B. C.; Frechet, J. M. J. L. Am. Chem. Soc. 2009, 131, 14160. 
(16) Huynh, W. U.; Dittmer, J. J.; Teclemariam, N.; Milliron, D. J.; Alivisatos, A. Phys. Rev. B 2003, 67, 115326.

(17) Breeze, A. J.; Schlesinger, Z.; Carter, S. A. Phvs. Rev. B 2001, 64 125205

(18) Van, D. J.; Yang, X.; Loos, J.; Bulle-Lieuwma, C. W. T.; Sievel, A. B.; Hummelen, J. C.; Janssen, R. A. I. Adv. Funct. Mater. 2004, 14, 425

(19) Hoppe, H.; Glatzel, T.; Niggemann, M.; Schwinger, W.; Schaeffler, F.; Hinsch, A.; Lux-Steiner, M.; Sariciftci, N. S. Thin Solid Films 2006, 511-512, 587.

(20) Hoppe, H.; Niggemann, M.; Winder, C.; Kraut, J.; Hiesgh, R. Hinsch, A.; Meissner, D.; Sariciftci, N. S. Adv. Funct. Mater. 2004, 14, 1005.

(21) Chou, S. Y.; Krauss, P. R.; Renstrom, P. L. Science 1996, 272 85.

(22) Voicu, N. E.; Ludwigs, S.; Crossland, E. J. W.; Andrew, P.; Steiner, U. Adv. Mater. 2007, 19, 757 .
(23) Kim, M.-S.; Kim, J.-S.; Cho, J. C.; Shtein, M.; Guo, L. J.; Kim, J. App. Phys. Lett. 2007, 90, 123113.

(24) Nanditha, D. M.; Dissanayake, M.; Adikaari, A. A. D. T.; Curry, R. J.; Hatton, R. A.; Silva, S. R. P. App. Phys. Lett. 2007, 90 , 253502

(25) Aryal, M.; Buyukserin, F.; Mielczarek, K.; Zhao, X.-M.; Gao, J.; Zakhidov, A.; Hu, W. I. Vac. Sci. Technol.. B 2008, 26, 2562.

(26) Ahn, S. H.; Guo, L. L. ACS Nano 2009, 3, 2304.

(27) McNeill, C. R.; Halls, J. J. M.; Wilson, R.; Whiting, G. L.; Berkebile, S.; Ramsey, M. G.; Friend, R. H.; Greenham, N. C. Adv. Funct. Mater. 2008, 18, 2309 .

(28) Hu, Z.; Baralia, G.; Bayot, V.; Gohy, J.-F.; Jonas, A. M. Nano Lett. 2005, 5, 1738

(29) Zheng, Z.; Yim, K.-H.; Saifullah, M. S. M.; Welland, M. E.; Friend, R. H.; Kim, J.-S.; Huck, W. T. S. Nano Lett. 2007, 7, 987.

(30) Marsh, R. A.; Groves, C.; Greenham, N. C. J. Appl. Phys. 2007, 101, No. 083509. 